LBNL-60662

\title{
Indoor exposure to radiation in the case of an outdoor release
}

\author{
Phillip N. Price, Buvana Jayaraman \\ Environmental Energy Technologies Division \\ Indoor Environment Department \\ Lawrence Berkeley National Laboratory \\ Berkeley, CA 94720
}

June 10, 2006

This work was supported in part by the Office of Chemical Biological Countermeasures, of the Science and Technology Directorate of the Department of Homeland Security, and was performed at Lawrence Berkeley National Laboratory through a work agreement with Lawrence Livermore National Laboratory. It was performed under U.S. Department of Energy Contract No. DE-AC02-05CH11231. 


\title{
Indoor exposure to radiation in the case of an outdoor release LBNL-60662
}

\author{
Phillip N. Price, Buvana Jayaraman \\ Environmental Energy Technologies Division \\ Lawrence Berkeley National Laboratory \\ Berkeley, CA 94720
}

June 10, 2006

\begin{abstract}
This report quantifies the effectiveness of "sheltering in place" in a commercial building in the event of an outdoor radiological release. The indoor exposure to airborne particles is calculated by solving the mass balance equation that accounts for the loss of particles due to deposition, filtration and exhaust. Quantitative estimates of shelter-inplace effectiveness are provided for typical commercial buildings.
\end{abstract}

\section{Acknowledgments}

This work was supported in part by the Office of Chemical Biological Countermeasures, of the Science and Technology Directorate of the Department of Homeland Security, and was performed at Lawrence Berkeley National Laboratory through a work agreement with Lawrence Livermore National Laboratory. It was performed under U.S. Department of Energy Contract No. DE-AC02-05CH11231.

\section{Introduction}

If airborne particles are released outdoors, they can be carried into a building through the Heating, Ventilating, and Air Conditioning (HVAC) system, through open windows and doors, and through cracks and penetrations through the building shell (a phenomenon known as "infiltration"). Several mechanisms remove particles from the indoor air: some particles will be exhausted by the HVAC system, some will be trapped in the building's air filters, and some will deposit in air ducts or on surfaces within the building. Particles deposited on indoor surfaces can "resuspend" into the air, by being kicked up by building occupants for example, and this can happen even long after the outdoor plume has dissipated. All of these factors help determine radiation exposure to building occupants in the event of an outdoor radiological release. Biwer et al. (2002) discuss the individual factors and their uncertainties in considerable detail.

In most commercial buildings, HVAC systems operate all the time, at least while the building is occupied, and 60 to 95 percent of the air that enters the building is provided by the HVAC system. HVAC systems normally have air filters, although the effectiveness of these filters varies widely due to differences in filter types and due to inadequate maintenance: some systems operate without filters or with some filters missing. 
Most commercial buildings recirculate some indoor air, at least in certain weather conditions, as a way to reduce heating and cooling costs. The recirculated air normally passes through the air filters, as does incoming air (see Figure 1).

HVAC systems are designed to provide air to all portions of a building, so even though commercial buildings can be very large and complicated, outdoor air (and any airborne pollutants that it carries) will often be spread effectively throughout the structure.

Figure 1 shows a schematic of the simplified building model that we consider in this report. Outdoor air, and contamination, enters the building through the HVAC system, passing through filters as it does so. Inside the building, some particles deposit (mostly but not exclusively onto horizontal surfaces), others are re-circulated through the air filter and some of these are captured by the filter, and some are exhausted from the building. Some of the deposited particles may resuspend, i.e. become airborne again, although as we discuss below this phenomenon turns out to be rather unimportant in determining indoor exposure to an outdoor airborne release.

This simplified view of building ventilation ignores some important phenomena, notably "bypass" around air filters in the HVAC system, and infiltration through windows, doors, and building cracks; we discuss the effect of these omissions in more detail later.

Very few data exist concerning the opening of windows and doors to provide ventilation in non-residential buildings; such behavior certainly varies with building type, location, and weather. As Figure 1 shows, we do not explicitly include the effects of open windows or of leakage through the building shell; the effect of these pathways is to decrease the effective filter efficiency. We discuss this in slightly more detail in a later section. For most large, urban non-residential buildings that are serviced by HVAC systems, ventilation is dominated by the HVAC system and we believe the results presented in this report can be directly used to estimate the relationship between indoor and outdoor exposures.

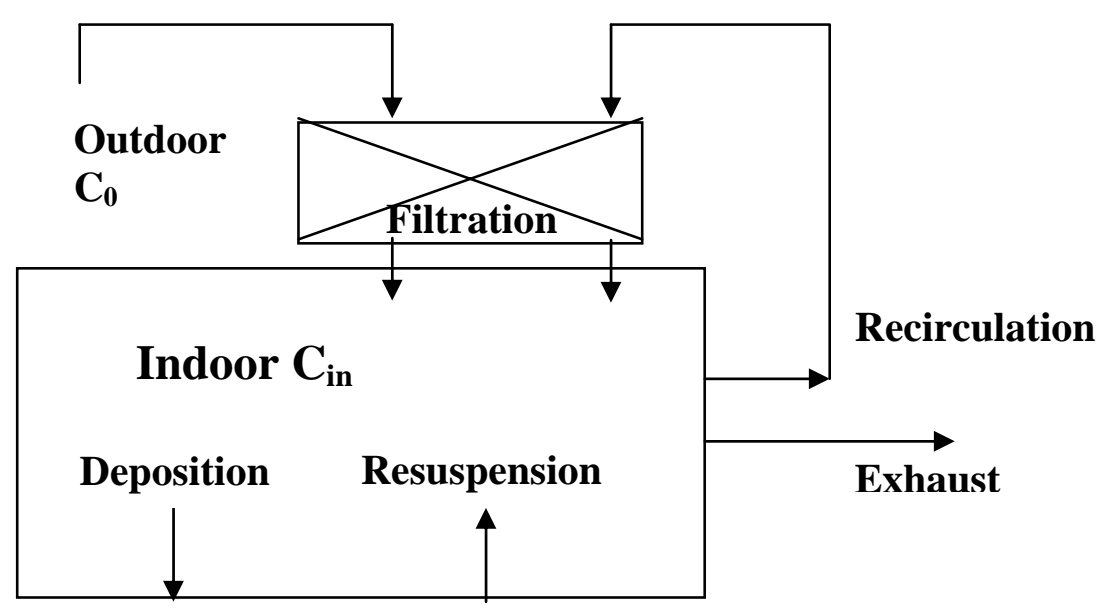


Figure 1 Schematic diagram showing the particle removal mechanisms that we consider in this report.

\section{Methods}

The following mass balance equation is used to estimate the exposure, accounting for infiltration, recirculation, deposition and filtration as indicated in Figure 1:

(2) $\frac{d D_{r}}{d t}=\frac{s_{r} C_{i}}{\sigma_{r}}-r D_{r}$

(3) $\frac{d D_{d}}{d t}=\frac{s_{d} C_{i}}{\sigma_{d}}$

where

$\mathrm{C}_{\mathrm{i}}$ : indoor concentration (particles $\mathrm{m}^{-3}$ )

$\mathrm{C}_{0}$ : outdoor concentration (particles $\mathrm{m}^{-3}$ )

Q: indoor-outdoor air exchange change rate $\left(\mathrm{h}^{-1}\right)$, equal to flow rate $\left(\mathrm{m}^{3} \mathrm{~h}^{-1}\right)$ divided by building volume $\left(\mathrm{m}^{3}\right)$

$\mathrm{F}$ : recirculated air change rate $\left(\mathrm{h}^{-1}\right)$

$f$ : filtration efficiency

$\mathrm{S}_{\mathrm{d}}$ : “permanent” deposition loss rate coefficient $\left(\mathrm{h}^{-1}\right)$

$\mathrm{s}_{\mathrm{r}}$ : "temporary" deposition loss rate to areas where resuspension may occur $\left(\mathrm{h}^{-1}\right)$

$\mathrm{r}$ : resuspension rate from "temporary" areas $\left(\mathrm{h}^{-1}\right)$

$D_{r}, D_{d}$ : deposition where resuspension is/isn't possible (particles $/ \mathrm{m}^{2}$ )

$\sigma_{r}$ : surface area where resuspension is possible, divided by volume of building $\left(\mathrm{m}^{2} / \mathrm{m}^{3}\right)$

$\sigma_{d}$ : surface area where deposition is permanent, divided by volume of building $\left(\mathrm{m}^{2} / \mathrm{m}^{3}\right)$

In Equations 1-3, all of the parameters depend on the size of the particles except for $\mathrm{Q}$ and $\mathrm{F}$.

\section{No resuspension}

We now consider the case of no resuspension $(r=0)$. In this case the system can be solved analytically. There is no deposition to locations where resuspension is possible, so $\mathrm{S}_{\mathrm{r}}=0$, but we will continue to carry this term in the equations, for future convenience. 
We first consider a "square-pulse" outdoor release: $\mathrm{C}_{0}>0$ between $\mathrm{t}=0$ and $\mathrm{t}=\mathrm{T}$, and $C_{0}=0$ otherwise. For the square-pulse release, the indoor concentration as a function of time can be determined analytically, as can the amount of contamination that deposits in the building. For $0<\mathrm{t}<\mathrm{T}$ :

$$
\begin{aligned}
& C_{i}(t)=C_{0} \frac{Q(1-f)}{\lambda}\left(1-e^{-\lambda t}\right) \\
& D_{d}(t)=C_{0} \frac{Q(1-f) s_{d}}{\lambda^{2} \sigma_{d}}\left[\lambda t+e^{-\lambda t}-1\right]
\end{aligned}
$$

where

$$
\lambda=Q+f F+s_{d}+s_{r}
$$

After the outdoor pulse ends at $\mathrm{t}=\mathrm{T}$ the indoor airborne particle concentration drops, since no new particles enter the building. Defining $\mathrm{C}_{\mathrm{T}} \equiv \mathrm{C}_{\mathrm{i}}(\mathrm{t}=\mathrm{T})$ we have:

$$
\begin{aligned}
& C_{i}(t)=C_{T} e^{-\lambda(t-T)} \\
& D_{d}(t)=\frac{C_{0} Q(1-f) S_{d}}{\lambda \sigma_{d}}\left(T+e^{-\lambda T}-e^{-\lambda(t-T)}\right)
\end{aligned}
$$

The resulting indoor exposure - that is, the time-integrated airborne concentration - up to $\mathrm{t}=\mathrm{T}$ is

$$
E_{1}=\int_{0}^{T} C_{i}(t) d t=C_{0} \frac{Q(1-f)}{\lambda^{2}}\left[\lambda T-1+e^{-\lambda T}\right]
$$

and for $\mathrm{t}=\mathrm{T}$ to $\mathrm{t}=\infty$ is

$$
E_{2}=\int_{T}^{\infty} C_{i}(t) d t=C_{0} \frac{Q(1-f)}{\lambda^{2}}\left(1-e^{-\lambda T}\right)
$$

The total indoor exposure is given by

$$
\text { (11) } \quad E_{1}+E_{2}=C_{0} \frac{Q(1-f) T}{\lambda}
$$

In contrast, for the square pulse the outdoor exposure is given by

$$
E_{\text {Outdoor }}=\int_{0}^{\infty} C_{0}(t) d t=C_{0} T .
$$

\section{Resuspension}

We now turn to the case that resuspension is possible. The system of equations can be solved analytically, although the solution is very complicated and we do not give it here. However, in a result that we will use later, we note that before $\mathrm{t}=\mathrm{T}$ (i.e. before the end of the outdoor pulse), if there is no resuspension then the ratio of deposited particles to airborne particles is given by 


$$
\frac{\sigma_{d} D_{d}(t)}{C_{i}(t)}=\frac{s_{d}}{\lambda}\left[\frac{\lambda t}{1-e^{-\lambda t}}-1\right]
$$

We define the "exposure factor," $\varepsilon \equiv E_{\text {in }} / E_{\text {out }}$. Multiplying the outdoor exposure by this factor will yield the indoor exposure; for instance, if $\varepsilon=0.5$, the indoor exposure is half the outdoor exposure. Combining Equations 11 and 12, the result is

$$
\varepsilon=\frac{Q(1-f)}{Q+f F+s_{d}+s_{r}}
$$

The exposure factor is independent of both the outdoor concentration and the duration of the pulse. By superposition, an outdoor pulse with varying concentrations can be built up as a series of square pulses (using a Green Function approach). Since Equation 14 applies for each individual pulse, it also applies for a superposition of them. Thus, for any outdoor concentration-vs.-time profile, Equation 13 applies.

The fraction of the exposure that occurs after the outdoor pulse has passed is given by $E_{2} /\left(E_{1}+E_{2}\right)$, which, for a square outdoor pulse, is equal to

$$
\frac{E_{2}}{E_{1}+E_{2}}=\frac{1-e^{-\lambda T}}{\lambda T}
$$

Since Equation 15 depends on $\mathrm{T}$, the superposition principle does not apply and Equation 15 will not hold exactly for a time-varying outdoor plume.

The exposure factor is important for determining the level of protection provided to building occupants by virtue of remaining inside the building rather than being outdoors. However, it considers only the exposure to airborne particles. Two other relevant issues are (1) how much contamination will remain on surfaces in the room, and (2) how much contamination will be trapped on the building's air filters, cracks in the building envelope, etc.? The amount of contamination that remains in the building is important both from a standpoint of determining the radiation dose from non-airborne particles and for characterizing decontamination requirements. Knowing the amount of contamination trapped by filters may help to rapidly estimate the level of contamination, since it will be easier to measure the amount of radioactivity in a filter than to survey an entire building. 
For a square pulse, the total number of particles of a given size that enter the building (before reaching the filters) is given by $C_{0} T Q$. There are three removal mechanisms: filtration, deposition, and exhaust, and all particles are eventually removed from the air by one of these mechanisms. (The number of particles that are deposited in people's lungs is a tiny fraction of the total number of particles, which we ignore). The relative importance of each removal mode is determined by the rate constant for that mode relative to the total of all of the rate constants, with the single complicating factor that some of the particles are removed by filtration before they enter the building. The result is that, as a fraction of the $C_{0} T Q$ particles that cross the building shell, the particle fates are as follows:

(16) Exhausted from building: $X=\frac{(1-f) Q}{Q+f F+s_{r}+s_{d}}$

$$
\text { Deposited: } \Delta=\frac{(1-f)\left(s_{r}+s_{d}\right)}{Q+f F+s_{r}+s_{d}}
$$

$$
\text { Trapped by filters or building envelope: } \phi=f+\frac{(1-f) f F}{Q+f F+s_{r}+s_{d}}
$$

As is true of the exposure factor, the fact that these expressions are independent of $C_{0}$ and $T$ implies that they hold for any release, not just a "square pulse" release.

\section{Filtration Efficiency}

The filtration efficiency, $f$, is determined by all of the factors other than deposition and exhaust that remove particles from the air. Therefore the filtration efficiency is not determined by the HVAC filters alone. Two effects in particular are significant:

1. Some air may bypass the filters (so that the filtration efficiency of the HVAC system, may be lower than implied by the filters alone), and

2. Some particles may enter the building directly, e.g. through cracks under doors and around windows, thus avoiding a first pass through the HVAC filters as they enter the building.

If we were to incorrectly assume that the filtration effectiveness $f$ is determined by the HVAC filters themselves, then the effect of phenomenon (1) is to decrease the value of $f$ in the denominator of Equation 14, while the effect of (2) is to decrease the value of $f$ in the numerator. The resulting value of $f$ will not, in general, be the same in the numerator and the denominator, so Equation 14 should be modified to read

$$
\varepsilon=\frac{Q\left(1-f_{o}\right)}{Q+f_{i} F+s_{d}+s_{r}}
$$

where $f_{o}$ is the filtration efficiency for particles entering the indoor air from outside (whether or not they pass through the HVAC filters, i.e. including the effects of 
penetration through the building shell, through windows, etc.), and $f_{i}$ is the filtration effectiveness for air that is re-circulated by the HVAC system, whether or not it passes through the HVAC filters. In both cases the filtration effectiveness is lower than would be implied by considering the effectiveness of the HVAC filters in isolation.

The separate filtration efficiency values, $f_{o}$ and $f_{i}$, will be highly correlated but not identical. They will be highly correlated because some substantial effects, such as filter bypass, will apply equally or almost equally to air entering from outdoors and to air that is recirculated. But they will not be identical because some effects, such as penetration of particles under exterior doors, will apply only to one term or the other. As a practical matter, attempting to quantify each of the filtration parameters separately, as a function of particle size, would add complexity without substantially reducing errors. Instead, throughout this report we apply Equation 14 but note that the filtration efficiency $f$ is the effective filtration efficiency of the HVAC system and the building envelope taken together, and not the HVAC filter efficiency.

Liu (2002) measured particle penetration through various building components (such as windows, cracks between bricks, etc.), and also determined approximate "penetration factors" (equal to1- $f$ ) for particle penetration into a single-family home. Liu's findings suggest that filtration efficiency is in the range $0.2-0.6$ for particles below $1 \mu \mathrm{m}$, and in the range $0.5-1$ for particles in the range $1-10 \mu \mathrm{m}$, and again in the range $0.2-0.6$ for particles larger than $10 \mu \mathrm{m}$.

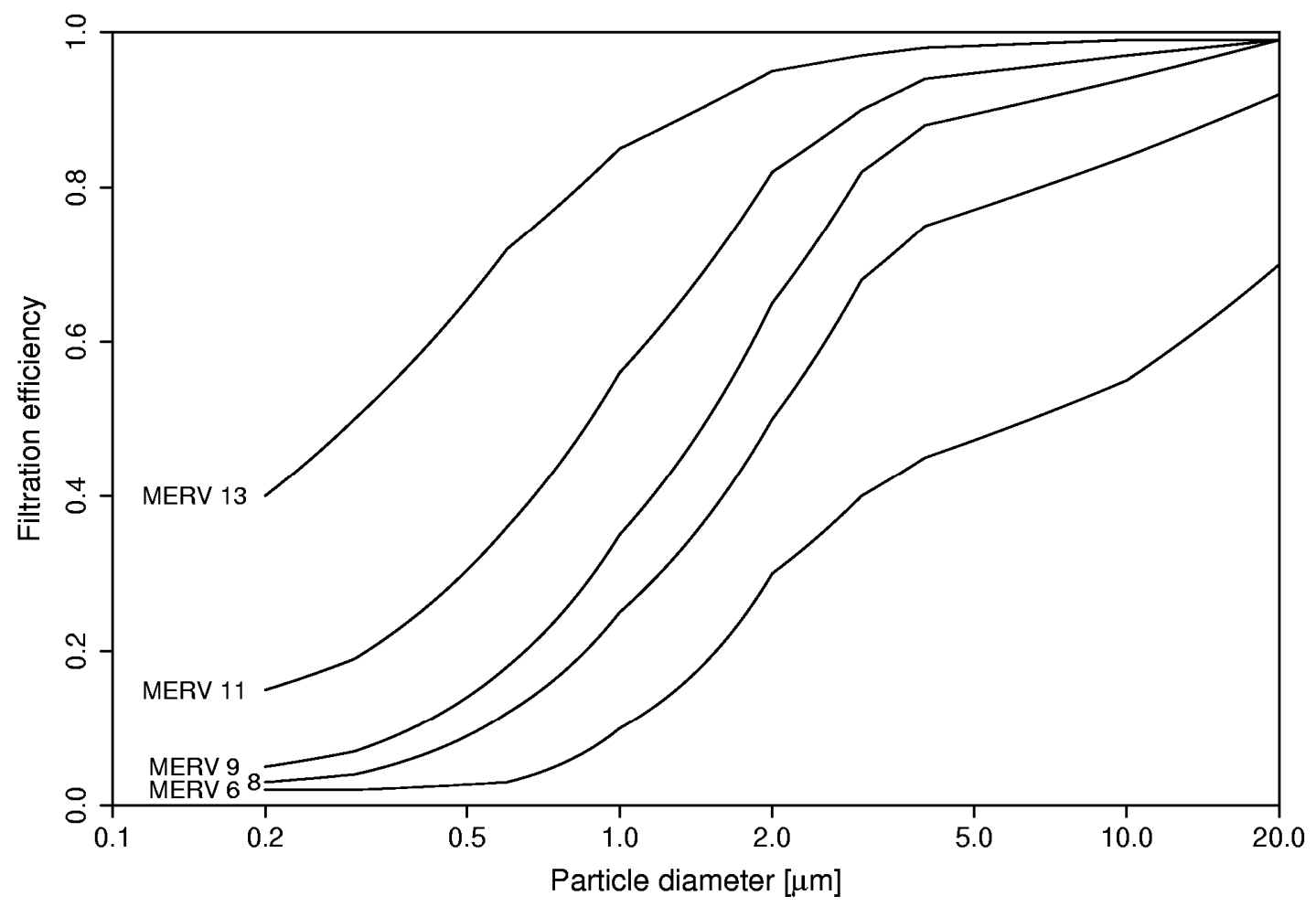


Figure 2 Filtration efficiency versus particle diameter, for filters with MERV rating of 6, $8,9,11$, or 13 . The $\mathrm{x}$-axis is logarithmic.

The American Society of Heating, Refrigerating, and Air Conditioning Engineers has defined standards for quantifying filter effectiveness (ASHRAE, 2000). A value called "Minimum Efficiency Reporting Value," or MERV, is assigned based on the effectiveness of the filter in various size ranges. MERV values exceeding 16 are equivalent to HEPA (High Efficiency Particulate Air) filters. Most buildings have filters in the MERV 6 to MERV 11 range. However, as previously discussed the HVAC filters alone do not determine the building's filtration effectiveness: A building that has very good filters, but has some filter bypass and some building envelope leakage, will act (when it comes to the exposure factor) similarly to a building that has mediocre filters but has no bypass or leakage. For example, a building that has "MERV 13" filters installed in its HVAC system may have a level of protection that is actually equivalent to only having MERV 11 or MERV 9 filtration.

In this report we consider five levels of filtration effectiveness: No filtration, MERV 6 (equivalent to ASHRAE Dust spot 30\% filter), MERV 9 (equivalent to ASHRAE Dust spot 45\% filter), MERV 11 (equivalent to ASHRAE Dust spot 65\% filter), and MERV 13 (equivalent to ASHRAE Dust spot 85\% filter). There is no single filter efficiency curve (i.e. efficiency as a function of particle size) for a given MERV rating: the curve can vary from manufacturer to manufacturer. Also, as time passes the filter efficiency tends to increase, because particles block the filter so the gaps in the filter become smaller. In this report we use filter efficiency curves (Figure 2) that are similar to the curves reported in Fisk et al. (2001) and Kowalski et al. (1999), but the performance of any given filter may not match our presumed curve exactly.

\section{Deposition rate}

According to Vajda (2001), radioactive particles with diameter less than $1 \mu \mathrm{m}$ are rarely produced explosively. Particles larger than $20 \mu \mathrm{m}$ settle out of the air very quickly and are also removed effectively even by very low-quality filters. For these reasons, we consider particles in the range 0.2 to $20 \mu \mathrm{m}$, which is wider than the range that we believe to be most relevant. The exposure factor for particles substantially larger than $20 \mu \mathrm{m}$ decreases very rapidly with particle size, and is less than 0.02 for $40 \mu \mathrm{m}$ particles even in the absence of filtration, due to the extremely high settling rate of these large particles.

The deposition rate for a given particle size reported in literature varies by an order of magnitude (Howard-Reed et al., 2003; Thatcher et al., 2001), and there is no single theoretical model that convincingly determines the behavior. This is especially true for ultrafine particles (diameter much less than $1 \mu \mathrm{m}$ ), for which the deposition rate is strongly affected by turbulence, electrostatic effects, surface roughness, etc., and for which experiments are hard to perform. For the range $1-10 \mu \mathrm{m}$ the deposition rates used in the current study are based on the experiments conducted by Thatcher et al. 
(2002) in a $2.2 \mathrm{~m} \mathrm{x} 2.7 \mathrm{~m} \times 2.4 \mathrm{~m}$ room; these fall more or less in the middle of rates reported in the literature. For particles smaller than $1 \mu \mathrm{m}$ we used a linear relationship between deposition rate and particle diameter that approximately fits the central envelope of reported deposition rates from a large number of studies summarized in Howard-Reed et al. (2003).

Thatcher reports deposition rates corresponding to a quiescent room with bare walls, and a fully furnished room with a room velocity of $5 \mathrm{~cm} / \mathrm{s}$, and finds substantial differences (about a factor of two) in deposition rate for these differing conditions for 1 $\mu \mathrm{m}$ particles. As shown in Table 1 and in the Appendix, empirically the deposition rate, $s$ $\left[h^{-1}\right]$ for either velocity condition can be predicted reasonably well with the equations:

$$
\begin{aligned}
& \text { (20) Diameter }>1 \mu \mathrm{m}, \quad s=0.2 d^{1.6} \\
& \text { (21) Diameter }<1 \mu \mathrm{m}, \quad s=0.2 d
\end{aligned}
$$

where $\mathrm{d}$ is the particle diameter in $\mu \mathrm{m}$. These equations agree within approximately a factor of three with the results of most other experiments that explored a wider range of particle sizes; further discussion is in the Appendix. The deposition rate for very small particles is extremely uncertain. For instance, published values for $0.35 \mu \mathrm{m}$ particles range from $0.05 \mathrm{~h}^{-1}$ to $0.7 \mathrm{~h}^{-1}$.

Table 1: Deposition rate as a function of particle diameter, as measured by Thatcher et al. (2002), and from an empirical fit (right column).

\begin{tabular}{|l|r|r|l|}
\hline $\begin{array}{l}\text { Particle } \\
\text { diameter } \\
(\mu \mathrm{m})\end{array}$ & $\begin{array}{l}\text { Deposition rate }\left(\mathrm{h}^{-1}\right) \\
\text { Fan off } \\
\text { Bare room surfaces }\end{array}$ & $\begin{array}{l}\text { Deposition rate }\left(\mathrm{h}^{-1}\right) \\
\mathrm{V}=5 \mathrm{~cm} / \mathrm{s} \\
\text { Fully furnished room }\end{array}$ & $\begin{array}{l}\text { Predicted } \\
\text { rate }\left[\mathrm{h}^{-1}\right] \\
=0.2^{*} \text { size }[\mu \mathrm{m}]^{\wedge} 1.6\end{array}$ \\
\hline 1 & 0.13 & 0.28 & 0.20 \\
\hline 2 & 0.58 & 0.83 & 0.61 \\
\hline 4 & 2.3 & 2.15 & 1.8 \\
\hline 8 & 6.1 & 5.2 & 5.6 \\
\hline
\end{tabular}

\section{Results}

\section{Exposure Factor}

We use exposure factor to quantify the effectiveness of sheltering in place: exposure factor compares the time-integrated airborne particle concentration that someone would experience if they remain indoors to what they would experience if they were outdoors. An exposure factor of 0.5 means that remaining indoors leads to half the exposure that would be experienced outdoors.

Exposure factors are shown in Figure 3. Each of the four sub-plots presents a different combination of indoor-outdoor air change rate (Q, in building volumes per hour), and a different rate of recirculation of air through filters ( $\mathrm{F}$, in building volumes per hour). Total air change rates shown range from $0.75 \mathrm{ACH}$ to $6.0 \mathrm{ACH}$, a range that Thatcher et 
al. (2001) found to cover most commercial buildings in the U.S. The upper plots consider the case that $20 \%$ of the air is recirculated into the building via the HVAC filters, while the lower plots assume $60 \%$ recirculation. These values are typical, but higher or lower recirculation

For most buildings, and for particle sizes less than $5 \mu \mathrm{m}, \lambda$ values (Equation 6) are in the range 1 to $8 \mathrm{~h}^{-1}$. From Equation 15 we see that in the case of a short-duration outdoor particle plume, most of the exposure will occur after the outdoor plume has passed. For instance, if $\lambda=3 \mathrm{~h}^{-1}$, and an outdoor plume of 12 minute (0.2-hour) duration, $75 \%$ of the exposure will occur after the outdoor plume has passed.

If there is no filtration $(f=0)$ then Equation 14 reduces to

$$
\text { (22) If } f=0: \varepsilon=\frac{1}{1+\left(s_{d}+s_{r}\right) / Q}
$$

so the "no filtration" curve is the same in both of the plots on the left side in Figure 3 (since they both have $Q=0.6 \mathrm{~h}^{-1}$ ) and in both of the plots on the right side of the figure $\left(Q=2.4 \mathrm{~h}^{-1}\right)$. 
$Q=0.6, F=0.15: 0.75 \mathrm{ACH}, 20 \%$ recirc.

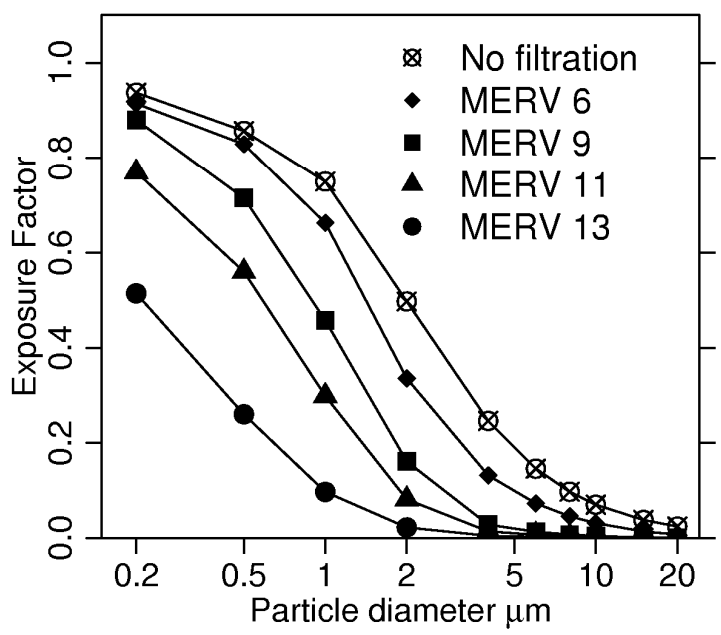

$Q=0.6, F=0.9: 1.5 \mathrm{ACH}, 60 \%$ recirc.

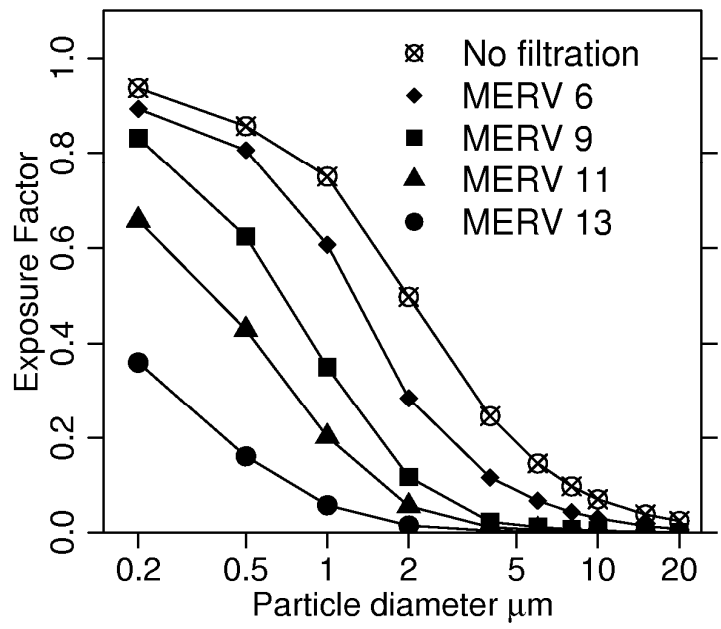

$Q=2.4, F=0.6: 3.0 \mathrm{ACH}, 20 \%$ recirc.

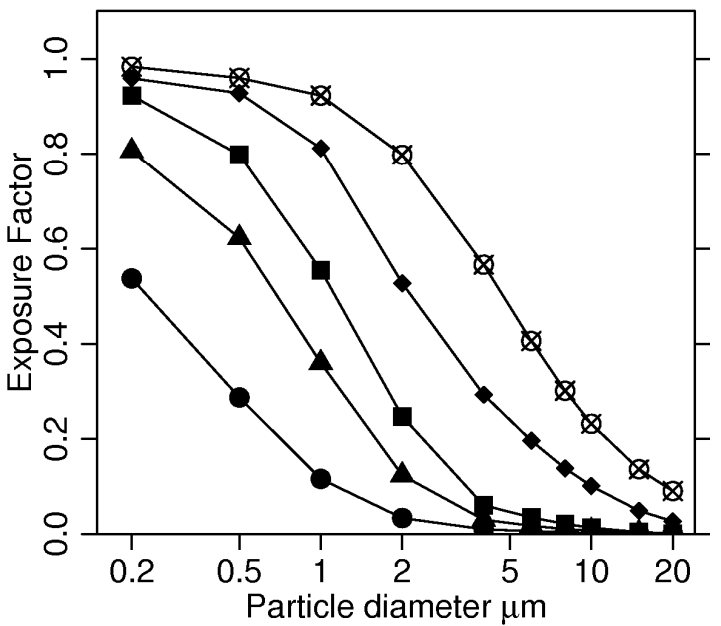

$Q=2.4, F=3.6: 6.0 \mathrm{ACH}, \mathrm{f}=60 \%$ recirc.

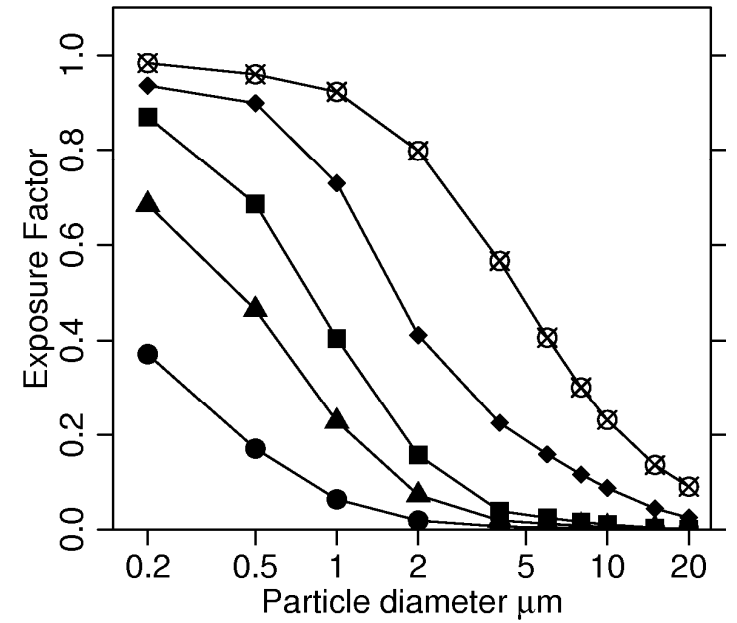

Figure 3 Exposure factor versus particle size, for different filtration levels. Filtration level corresponds to the effective filtration of the entire building, which will generally be lower than implied by the HVAC filters alone. Each plot shows a different combination of indoor-outdoor air change rate $\left(\mathrm{Q} \mathrm{h}^{-1}\right)$ and recirculation rate $\left(\mathrm{F} \mathrm{h}^{-1}\right)$, and thus a different combination of total air changes per hour $(\mathrm{ACH})$ and recirculation fraction, as indicated in the plot titles. $\mathrm{X}$-axis is logarithmic.

\section{Particle fate}

Figure 4 shows the eventual fate of the particles, as determined from Equations 16-18, for several different filtration performance levels but for the same building operating conditions: $\mathrm{Q}=2.4 \mathrm{~h}^{-1}$ and $\mathrm{F}=0.6 \mathrm{~h}^{-1}$ or, equivalently, $3.0 \mathrm{ACH}$ and $20 \%$ recirculation. The fractions deposited, exhausted, and removed by filtration add to unity for all particle sizes. As discussed above, "filtration performance" refers to the effective 
filtration for the entire building, including the effects of filter bypass and air infiltration that does not pass through the filters.

The curves are qualitatively similar for different levels of $\mathrm{Q}$ or $\mathrm{F}$ from those shown here. Particles smaller than about $0.5 \mu \mathrm{m}$ in diameter deposit very slowly and are not removed efficiently by filters, so they remain airborne for a long time and are eventually expelled from the building by the HVAC system. In contrast, particles larger than $5 \mu \mathrm{m}$ deposit quickly and are effectively removed by filters; small cracks in the building shell also act as filters for these particles when air infiltrates the building. As a result, deposition and filtration both remove most of these particles before they are exhausted by the HVAC system. In the intermediate size range, there is no dominant removal mechanism for particles between 0.5 and $5 \mu \mathrm{m}$ in diameter. 

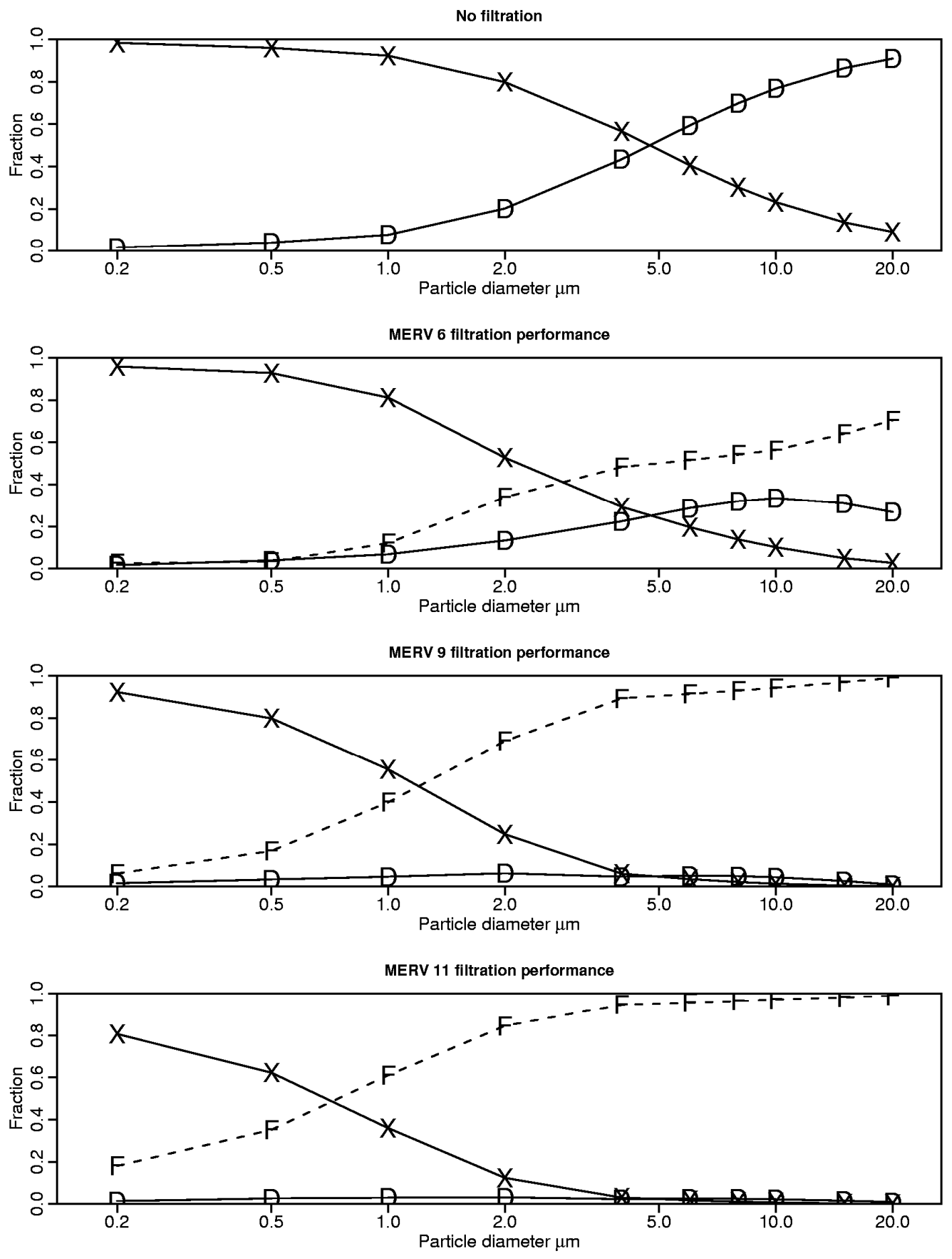

Figure 4 Fate of particles for 4 levels of filtration: fraction of particles as a function of particle diameter. $\mathrm{X}=$ exhausted from the building, $\mathrm{D}=$ deposited within the building, $\mathrm{F}=$ trapped in HVAC filters or building envelope. All cases show $\mathrm{Q}=2.4 \mathrm{~h}^{-1}, \mathrm{~F}=0.6 \mathrm{~h}^{-1}$, which corresponds to $3 \mathrm{ACH}$ with $20 \%$ recirculation. Filtration level corresponds to the effective filtration of the entire building, which will generally be lower than implied by the HVAC filters alone. X-axis is logarithmic. 


\section{Resuspension}

Equation 13 gives the ratio of deposited particles to airborne particles before the outdoor particle pulse has ended, in the case of a "square" outdoor pulse, if there is no resuspension. Consider a case with no HVAC filtration $(f=0)$, and $Q=2.5 \mathrm{~h}^{-1}$. Figure 5 shows the ratio of airborne to deposited particles as a function of particle diameter for various pulse durations.

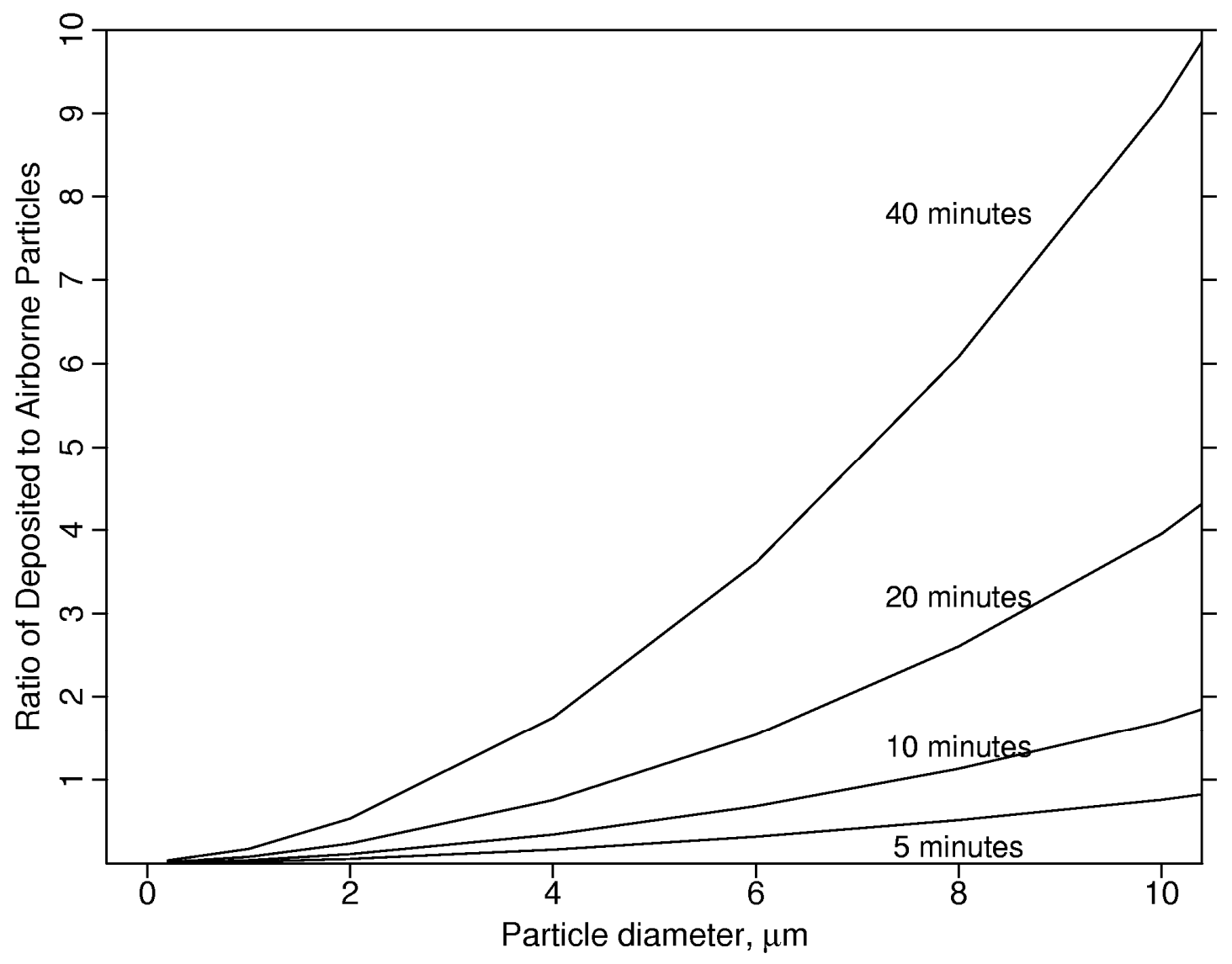

Figure 5: Ratio of deposited to airborne particles, as a function of diameter, at the moment that the outdoor square pulse release ends. Each curve represents a different duration of outdoor pulse.

As Figure 5 shows, at the moment the outdoor pulse ends, the total number of particles deposited in the building can be anywhere between 0.01 times and approximately 10 times the total number of airborne particles. If, at that moment, $1 \%$ of the deposited particles of each size were to become airborne, the effect would range from negligible (a less than $1 \%$ increase in the number of airborne small particles) to small (up to a $10 \%$ increase in the number of airborne large particles). Prior to this moment, the ratio of deposited to airborne particles is even smaller. 
As this example illustrates, resuspension will provide only a very small contribution to airborne concentrations, and thus to exposure, until after the outdoor plume has passed. After the plume has passed, and after almost all of the remaining airborne particles have either deposited or been exhausted from the building - that is, after a time $>1 / \lambda$ - resuspension will be a significant effect because it will be the only effect that contributes to airborne exposure.

Experiments (not yet published) by Mark Sippola and others at Lawrence Berkeley National Laboratory suggest that each minute of disturbance of a surface on which particles have been deposited will resuspend between $0.1 \%$ and $1 \%$ of the particles on the surface, with only a weak dependence on the size of the particles. (However, a large particle, even if resuspended, may not rise high enough to reach the breathing zone of people, and will quickly redeposit.) "Disturbance" in this case refers to either direct physical contact of an object with the surface - e.g. stepping on a carpet - or shaking or moving a surface. In a typical building, only a small fraction of total surface area is likely to be disturbed in this way in a single minute, hour, or even day: the tops of books in bookshelves, the edges and corners of rooms, the floors of unoccupied rooms, the tops of ceiling tiles, the floor under and behind desks, and so on, add up to a very large area that will remain largely undisturbed. In fact, in a one-hour period of normal building use we might expect only $0.5 \%$ to $5 \%$ of a building's total surface area to be directly contacted or moved, and some of this area will be disturbed only for a few minutes. If we assume that $3 \%$ of the horizontal surface area in a building is disturbed at some point within a 1-hour period, that the disturbance of a given location lasts for 10 minutes on average, and that $0.5 \%$ of particles are resuspended for each minute of disturbance, then we estimate that $0.15 \%$ of deposited particles will resuspend at some point within the hour. This estimate is uncertain within at least a factor of ten in either direction, so our estimated range is $0.015 \%$ to $1.5 \%$. Even the largest of these represents a very small contribution to the total airborne concentrations until at least a few hours after the plume has passed.

\section{Discussion}

This report has considered the case that a building's HVAC system is operated throughout a radiological event, so that the building ingests particles once the outdoor plume arrives, and expels them after the contaminated plume passes. If, instead, the HVAC system is shut off after the plume arrives, indoor exposures could actually be higher than outdoor exposures, because some contamination will be trapped in the building that would otherwise be exhausted or would be trapped by filters during recirculation.

As this report shows, sheltering in buildings provides some protection from airborne particles in an outdoor plume. Buildings provide substantial protection for particles larger than $5 \mu \mathrm{m}$ in diameter, with exposure (i.e. time-integrated airborne particle concentration) reduced by at least a factor of 3 compared to outdoor conditions. Protection is even greater for particles $10 \mu \mathrm{m}$ in diameter, with exposures reduced by a factor of 5 or more compared to outdoors. As expected, protection levels are highest when the indoor-outdoor air exchange rate is low and when effective filters are used; in these cases, exposure factors of 0.01 to 0.1 can be achieved for particles larger than $5 \mu \mathrm{m}$ 
in diameter; that is, indoor exposures are reduced by a factor of 10 to 100 compared to outdoors.

For a short-duration outdoor particle plume, much of the indoor exposure will occur after the outdoor plume has dissipated. This occurs because some of the airborne contamination that enters the building is trapped in the building for a long time. Prompt evacuation after the outdoor plume has dissipated could substantially reduce exposures. However, the real-world case of time-varying (rather than square-pulse) outdoor exposures, combined with the possibility of resuspension of outdoor particles, will probably lead to prompt evacuation being somewhat less important than these results imply.

As discussed previously, filtration effectiveness cannot be determined from the HVAC filter type alone, for two reasons: (1) filters are usually installed with gaps around them so that air can bypass them, and some filters may be missing, and (2) if the HVAC system does not maintain the building at a higher pressure than the outdoors, some particles will enter through the building envelope and thereby bypass the filters. A building that nominally has MERV-13 filters - an unusually high level of filtration, might only attain the level of protection implied by the MERV-11 or MERV-9 curves on the plots. For a typical commercial building with an operating HVAC system, closed doors and windows, and typical filters and leakage characteristics, the exposure factor is likely to fall in the range between "no filtration" and "MERV 9" on the curves in Figure 3. Thus, a typical commercial building may experience an exposure factor somewhere in the range $0.4-0.9$ for $1 \mu \mathrm{m}$ particles, $0.05-0.4$ for $5 \mu \mathrm{m}$ particles, and $0.02-0.2$ for $10 \mu \mathrm{m}$ particles.

Resuspension turns out to provide a negligible contribution to airborne exposure to anyone who is in the building during the passage of the outdoor plume. However, after the plume has dissipated and the indoor particles have either deposited or been exhausted from the building, resuspension may cause non-negligible airborne exposure if a building is re-occupied (for instance, by emergency personnel searching for victims). A substantial level of activity (equivalent to normal use) within a contaminated building might resuspend $0.015 \%$ to $1.5 \%$ of the deposited particles; the effect would be somewhat comparable to another outdoor release of $0.015 \%$ to $1.5 \%$ the size of the actual release. More detailed analysis of the effects of resuspension could be carried out by choosing appropriate values for the resuspension terms in Equations 1-3, and using the resulting (complicated) analytical solutions, or finding numerical solutions to those equations. Given the results discussed above, it is doubtful that this would be worthwhile, especially before better estimates of resuspension rates are available.

This report has only considered the exposure to airborne particles, which are subject to inhalation. Discussion of source characteristics, such as the radiation activity in a given particle size range that might be expected from a "dirty bomb," is outside the scope of this report. 


\section{References:}

ASHRAE Standard 52.2: Method of Testing General Ventilation Air-Cleaning Devices for Removal Efficiency by Particle Size, American Society of Heating, Refrigerating and Air-Conditioning Engineers, 2000.

Biwer BM, Kamboj S, Arnish J, Yu C, and Chen SY. Technical basis for calculating radiation doses for the building occupancy scenario using the probabilistic RESRADBUILD 3.0 code. Argonne National Laboratory ANL/EAD/TM/02-1, 2002.

Fisk, W. J., Faulkner, D., Palonen, J., and Seppanen, O. Performance and costs of particle air filtration technologies. LBNL-47833. 2001.

Howard-Reed, C., Wallace, L. and Emmerich, S. J.: Deposition rates of fine and coarse particles in residential buildings: Literature review and measurements in an occupied townhouse, NISTIR 7068, October 2003.

Kowalski, W. J., Bahnfleth, W.P., \& Whittam, T.S. Filtration of airborne microorganisms: Modeling and prediction. ASHRAE Transactions, 105, 4-17, February 1999.

Liu, D-L. Air Pollutant Penetration through Airflow Leaks into Buildings. Lawrence Berkeley National Laboratory report LBNL-51498, 2002.

Thatcher, T. L., McKone, T. E., Fisk, W. J., Sohn, M. D., Delp, W. W., Riley, W. J. and Sextro, R. G.: Factors affecting the concentration of outdoor particles indoors (COPI): Identification of data needs and existing data. Lawrence Berkeley National Laboratory report LBNL-49321, December 2001.

Thatcher, T. L., Lai, A. C. K., Moreno-Jackson, R, Sextro, R. G. and Nazaroff, W. W.: Effects of room furnishings and air speed on particle deposition rate indoors, Atmospheric Environment, 36, 1811-1819, 2002.

Vajda N., Radioactive Particles in the Environment: Occurrence, Characterization, Appropriate Analytical Techniques. International Atomic Energy Agency, 2001. Obtained through International Atomic Energy Agency website. 


\section{Appendix: Calculation of Exposure Factor}

The ratio of indoor time-integrated particle concentration to outdoor time-integrated particle concentration is called the "exposure factor," $\varepsilon$. If resuspension is ignored, exposure factor is calculated with the equation:

(1) $\varepsilon=\frac{Q(1-f)}{Q+f F+s_{d}}$

where

- $\mathrm{Q}$ is the indoor-outdoor air exchange rate,

- $\mathrm{F}$ is the recirculation rate (i.e. the amount of air that is sent from the building, through the HVAC system, and back into the building),

- $f$ is the filtration efficiency (i.e. the fraction of particles that are removed by a single pass through the HVAC system); due to filter bypass and other factors, this efficiency will be lower than the efficiency of the HVAC filters themselves.

- $S_{d}$ is the particle deposition rate.

Equation (1) assumes that all of the parameters are constant - e.g. the ventilation system operation is not changed.

$\mathrm{Q}$ and $\mathrm{F}$ are independent of particle size; $f$ and $s_{d}$ are size-dependent.

Results of Turk et al. (1989), Lagus (1995), and Persily (1989) suggest that for most commercial buildings $\mathrm{Q}$ is in the range $0.8-2$. The fraction of air that is recirculated (equal to $\mathrm{F} /(\mathrm{Q}+\mathrm{F})$ ) is typically in the range $0.2-0.6$, although this ratio is 0 for some buildings and can be as high as 0.9 for some buildings in certain operating conditions.

Table A-1 reports "filter curves" - filtration efficiency as a function of particle size - for several levels of filtration. These curves approximate the filter curves in Kowalski et al. and Fisk et al. As discussed in the body of the report, most commercial buildings have filters in the MERV 6 to MERV 11 range, but the effective filtration level will be lower. The effective filtration for most buildings is probably somewhere in the range from no filtration (0 efficiency) to MERV 9.

Table A-2 reports deposition rates from Thatcher et al. (2002), Howard-Reed et al. (2003), and Wallace et al. (1997), as well as predictions from the equations:

(2) Diameter $>1 \mu \mathrm{m}, \quad s=0.2 d^{1.6}$

(3) Diameter $<1 \mu \mathrm{m}, s=0.2 d$ 
Table A-1: Assumed filtration efficiency as a function of MERV level and particle size.

\begin{tabular}{|l|c|c|c|c|c|c|c|c|c|}
\hline $\begin{array}{c}\text { MERV } \\
\text { level }\end{array}$ & \multicolumn{10}{c|}{ Particle Diameter $(\mu \mathrm{m})$} \\
& 0.2 & 0.3 & 0.6 & 1 & 2 & 3 & 4 & 10 & 20 \\
\hline 6 & 0.02 & 0.02 & 0.03 & 0.10 & 0.30 & 0.40 & 0.45 & 0.55 & 0.70 \\
\hline 9 & 0.05 & 0.07 & 0.18 & 0.35 & 0.65 & 0.82 & 0.88 & 0.94 & 0.99 \\
\hline 11 & 0.15 & 0.19 & 0.36 & 0.56 & 0.82 & 0.90 & 0.94 & 0.97 & 0.99 \\
\hline 13 & 0.40 & 0.50 & 0.72 & 0.85 & 0.95 & 0.97 & 0.98 & 0.99 & 0.99 \\
\hline
\end{tabular}

Table A-2: Deposition rate $\left(\mathrm{h}^{-1}\right)$ as a function of particle diameter. Experiment A1: Thatcher et al. 2002, fans off, bare room. A2: Thatcher et al. (2002), air moving $5 \mathrm{~cm} / \mathrm{s}$, furnished room. B: Howard-Reed et al. (2003), fans off. C: Wallace et al. [10]. P: Predicted deposition from Equations 2 and 3. Interpolation or extrapolation were performed where necessary, using a power-law function from nearby data points; extrapolation was performed for at most one bin of particle size outside the range of the measurements.

\begin{tabular}{|l|l|l|l|l|l|l|l|r|r|}
\hline Experiment & \multicolumn{10}{|c|}{ Particle Diameter $(\mu \mathrm{m})$} \\
& 0.2 & 0.3 & 0.6 & 1 & 2 & 3 & 4 & 10 & 20 \\
\hline A1 & & & 0.04 & 0.13 & 0.58 & 1.30 & 2.30 & 8.4 & \\
\hline A2 & & & 0.13 & 0.28 & 0.83 & 1.33 & 2.15 & 6.9 & \\
\hline B & & 0.34 & 0.40 & 0.45 & 0.90 & 1.10 & 1.50 & 8.0 & \\
\hline C & & 0.18 & 0.31 & 0.44 & 0.66 & 0.78 & 0.94 & 2.8 & \\
\hline P & 0.04 & 0.06 & 0.12 & 0.20 & 0.61 & 1.15 & 1.84 & 8.0 & 24 \\
\hline
\end{tabular}

\section{References for Appendix:}

ASHRAE Standard 52.2: Method of Testing General Ventilation Air-Cleaning Devices for Removal Efficiency by Particle Size, American Society of Heating, Refrigerating and Air-Conditioning Engineers, 2000.

Fisk, W. J., Faulkner, D., Palonen, J., and Seppanen, O. Performance and costs of particle air filtration technologies. LBNL-47833. 2001.

Howard-Reed, C., Wallace, L. and Emmerich, S. J.: Deposition rates of fine and coarse particles in residential buildings: Literature review and measurements in an occupied townhouse, NISTIR 7068, October 2003.

Kowalski, W. J., Bahnfleth, W.P., \& Whittam, T.S. Filtration of airborne microorganisms: Modeling and prediction. ASHRAE Transactions, 105, 4-17, February 1999. 
Thatcher T. L., Lai A. C. K., Moreno-Jackson, R, Sextro, R. G. and Nazaroff, W. W.: Effects of room furnishings and air speed on particle deposition rate indoors, Atmospheric Environment, 36, 1811-1819, 2002.

Wallace L.A., Quackenboss J., and Rodes C.: Continuous measurements of particles, $\mathrm{PAH}$, and $\mathrm{CO}$ in an occupied townhouse in Reston, VA. In: Measurement of Toxic and Related Air Pollutants, Proceedings of EPA-AWMA Symposium on Toxic and Related Compounds, Research Triangle Park, North Carolina, April 29-May 1, 1997, pp. 860871. Air and Waste Management Association, Pittsburgh, Pennsylvania. 